\title{
INVESTIGACIÓN
}

\section{NIVEL DE RIESGO OBSTÉTRICO EN MUJERES EMBARAZADAS QUE ACUDEN A UN CENTRO DE PRIMER NIVEL DE ATENCIÓN}

OBSTETRIC RISK LEVEL IN PREGNANT WOMEN ATTENDING TO A FIRST LEVEL ATTENTION CENTER

OLGA BARRAGÁN HERNÁNDEZ* JESÚS ANTONIO CRUZ QUINTERO** LUIS ARTURO MALDONADO LÓPEZ**, ALEJANDRA FAVELA OCAÑO*， SANDRA LIDIA PERALTA PEÑA ${ }^{* * *}$

\section{ABSTRACT}

Introduction: high levels of maternal mortality present in developing countries make it possible to define maternity at risk as the possibility that a woman or the product of suffering damage, injury or even death during gestation, delivery and puerperium processes.

Objective: to identify the obstetric risk level in pregnant women attending prenatal consultation to a fist level care center at Hermosillo, Sonora.

Methodology: descriptive, cross-sectional study. Population was integrated by pregnant women attending prenatal consultation during January-March of 2016 period. Sampling was for convenience and the sample was 145 pregnant women. The "Obstetric Risk Classification (CRO)" was used as measuring instrument.

Results: $22.1 \%$ is 18 years old or less. Women between 19 to 25 are the ones that presented the higher obstetric risk $(12.4 \%)$, in comparison with the teenagers between 18 and less (4.1\%).

Conclusions: Frequency and quality of prenatal control allows to make integral assessment of the mother-child binomial, contributing to early detection of factors that complicate pregnancy, delivery and puerperium, and may put at risk their lives.

Key Words: pregnancy high risk, risk factors, prenatal care (DeCS, BIREME).

\section{RESUMEN}

T ntroducción: Los elevados índices de mortalidad materna que se presentan en los países en desarrollo permite definir la maternidad en riesgo como la posibilidad que tiene una mujer o el producto de sufrir daños, lesión o incluso muerte durante el proceso de gestación, parto y puerperio.

Objetivo: identificar el nivel de riesgo obstétrico en mujeres embarazadas que acuden a consulta prenatal a un centro de primer nivel de atención en la ciudad de Hermosillo, Sonora.

Metodología: Estudio descriptivo, transversal. La población estuvo conformada por embarazadas que acudieron a consulta prenatal durante el período de enero-marzo del 2016. El muestreo fue por conveniencia y la muestra fue de 145 gestantes. Se utilizó como instrumento de medición la "Clasificación del Riesgo Obstétrico (CRO)".

Resultados: El 22.1\% tiene 18 años y menos de edad. Las jóvenes entre 19 a 25 años son las que presentaron mayor riesgo obstétrico $(12.4 \%)$, comparado con las adolescentes entre 18 años y menos (4.1\%).

Conclusiones: La frecuencia y calidad del control prenatal permite realizar evaluación integral de salud del binomio madre-hijo, contribuyendo a la detección temprana de factores que compliquen el embarazo, parto y puerperio, y que puedan poner en peligro su vida.

Palabras clave: embarazo, embarazo de alto riesgo, factores de riesgo, atención prenatal (DeCS, BIREME).

\footnotetext{
* Maestra en Ciencias. Departamento de Enfermería. Universidad de Sonora.

Correo para correspondencia: barragan@enfermeria.uson.mx

** Licenciado en Enfermería. Egresado del Dpto. de Enfermería de la Universidad de Sonora.

*** Doctora en Ciencias de Enfermería. Departamento de Enfermería. Universidad de Sonora.
} 


\section{INTRODUCCIÓN}

"Embarazo de alto riesgo es aquel en el que se tiene la certeza o la probabilidad de estados patológicos o condiciones anormales concomitantes con la gestación y el parto que aumentan los peligros para la salud de la madre o del producto o bien cuando la madre procede de un medio socioeconómico precario" (1).

Existen diferentes factores que determinan dicho riesgo, entre los cuales se encuentran: los antecedentes de muerte fetal, aborto habitual, paridad > 5 hijos, nuliparidad, antecedente de aborto inducido, parto prematuro o bajo o alto peso al nacer, enfermedades agregadas, ruptura prematura de membranas, hemorragia de la segunda mitad del embarazo o después del parto, estado socioeconómico bajo, edad materna menor de 16 años o mayor de 34, hábito de fumar, entre otros. Es importante destacar que cuando estos factores se asocian, el riesgo se incrementa y dicho riesgo se encuentra estrechamente relacionado con la mortalidad materna ${ }^{(2)}$.

Dentro de las principales metas de salud a nivel mundial, la disminución de la tasa de mortalidad materna se considera una de las más importantes. La Organización Mundial de la Salud (OMS) (3) define la mortalidad materna como la muerte de una mujer durante su embarazo, parto o dentro de los 42 días después de su terminación, por cualquier causa relacionada o agravada por el embarazo, parto o puerperio o su manejo, pero no por causas accidentales.

De igual forma los elevados índices demográficos de mortalidad materna e infantil que se presentan en los países en desarrollo permite definir la maternidad en riesgo como "la posibilidad que tiene una mujer o el producto de su embarazo de sufrir daños, lesión o incluso muerte durante el proceso de reproducción, gestación, parto y puerperio, por lo que es muy importante que el embarazo sea planificado, deseado y que la madre esté en las mejores condiciones biológicas, psicológicas, sociales y económicas más adecuadas para garantizar recién nacidos sanos" (4).

La Sociedad Mexicana de Salud Pública en México menciona que en el país se presentan 2.1 millones de embarazos cada año y que en 250 a 300 mil de estos ocurren complicaciones, 30 mil mujeres quedan con secuelas obstétricas que las incapacitan permanentemente y fallecen anualmente en promedio 1,100 mujeres por esta causa ${ }^{(5)}$.

Por otro lado, en el estado de Sonora, el observatorio de mortalidad materna en México ${ }^{(5)}$ establece que la razón de mortalidad materna (RMM) para el 2013 fue de 40.2 por 100 mil nacidos vivos, mientras que la media nacional fue de 38.2 y que, aunque el año 2014 descendió a 33.7, por debajo del promedio nacional (38.9), sigue siendo alta comparada con otras entidades del país.

En los últimos años, se ha identificado la necesidad de estudiar los casos de morbilidad obstétrica, para complementar las investigaciones sobre mortalidad materna como parte de las acciones propuestas por la Organización Panamericana de la Salud (OPS) y la OMS para la disminución de la mortalidad materna en el 2015 (6).

Dado el panorama epidemiológico existente que involucra a la mujer embarazada, se vio la necesidad de realizar un estudio de investigación que tuviera como objetivo identificar factores de riesgo obstétrico en mujeres embarazadas que acuden a consulta prenatal en un centro de salud urbano de primer nivel atención.

\section{METODOLOGÍA}

El tipo de estudio fue descriptivo y transversal, la población estuvo conformada por todas aquellas mujeres embarazadas usuarias de un Centro de Salud de primer nivel, que acudieran a consulta prenatal en el período de enero a marzo del 2016. El muestreo fue no probabilístico por conveniencia, y se contó con una muestra total de 145 gestantes. Como criterio de inclusión se estableció a aquellas mujeres que desearan participar en el estudio de forma voluntaria.

El instrumento de recolección titulado "Clasificación de Riesgo Obstétrico" (CRO), validado y utilizado por la Secretaría de Salud (SS), ofrece posibilidad de registrar la calificación de cinco valoraciones de riesgo obstétrico con variables que permitieron clasificar el nivel de riesgo obstétrico que presentaba la gestante. En el primer apartado contempla la ficha de identificación que contienen datos sociodemográficos, en el segundo se consideran los antecedentes personales patológicos, mientras que en el tercero los antecedentes ginecobstétricos, existe otro dirigido al embarazo actual y uno más al examen clínico anormal. Cada una de las variables contenidas en los diversos apartados se le asigna una puntuación que va de 1 a 3 puntos (excepto la variable: 2 o más cesáreas, correspondiente al apartado de antecedentes ginecobstéricos con un valor de 4), considerándose para la calificación la sumatoria de las variables de los cinco apartados, que permiten clasificar de bajo riesgo la puntuación 
$<5$ puntos, y de alto riesgo > 6 puntos. El formato de valoración da la posibilidad de realizar a las mujeres embarazadas varias mediciones en distintos momentos durante su período de gestación.

La recolección de la información se llevó a través de una entrevista estructurada (CRO) que se aplicó a mujeres embarazadas que acudían a la consulta prenatal a un centro de primer nivel de atención ubicado al noroeste de la ciudad de Hermosillo, minutos previos a la consulta en el módulo de atención a fin de identificar factores de riesgo obstétrico.

La captura de datos se llevó a cabo en el programa estadístico IBM SPSS versión 20 durante los meses de abril y mayo del mismo año, para el procesamiento de datos se empleó la estadística descriptiva que permitió la caracterización de los sujetos del estudio a través de frecuencias y porcentajes.

Para este estudio se tomó en cuenta lo establecido en el Reglamento de la Ley General de Salud en materia de investigación para la salud ${ }^{(7)}$ en el artículo 13, donde se establece que prevalecerá el criterio del respeto a su dignidad y la protección de sus derechos y bienestar. Del artículo 14, fracción V, VI, y VIII y artículo 18, ya que se obtuvo consentimiento informado y por escrito del sujeto de investigación, en este caso dicho consentimiento fue proporcionado a las embarazadas antes de aplicar el instrumento de evaluación, con previa explicación del propósito de la investigación.

\section{RESULTADOS}

Del total de mujeres embarazadas que participaron en el estudio el $22.1 \%$ tiene 18 años y menos de edad, mientras que el $44.8 \%$ y $23.4 \%$ de ellas solo cursaron el nivel básico de escolaridad, primaria y secundaria respectivamente. Respecto a sí cuentan con pareja o no el 30.3\% expreso no contar con su pareja que pudiera apoyarlas durante el período del embarazo, parto y puerperio (Tabla 1).

En cuanto a los antecedentes personales patológicos (Tabla 2) el $25.5 \%$ las participantes en el estudio comentaron que habían tenido cirugía pélvica/uterina, mientras que solo el $6.9 \%$ presentaban una enfermedad crónica no transmisible como la diabetes mellitus o hipertensión arterial. Por otro lado, la Tabla 3 muestra los signos de alarma o riesgo obstétrico que con mayor frecuencia se observaron en las mujeres embarazadas participantes en el estudio. El síntoma que mayormente manifestaron las gestantes fue la cefalea con un $20.7 \%$, seguida de edema en miembro pélvicos (10.3\%), malnutrición u obesidad (10.3\%), disuria (6.2\%) y anemia (6.2\%).
TABLA 1. DATOS SOCIODEMOGRÁFICOS DE LAS EMBARAZADAS

\begin{tabular}{|l|c|c|}
\hline VARIABLES & $\boldsymbol{f r}$ & $\mathbf{\%}$ \\
\hline Edad & 32 & $\mathbf{2 2 . 1}$ \\
18 y menores & 66 & 45.5 \\
$19-25$ & 21 & 14.5 \\
$26-30$ & 20 & 13.8 \\
$31-35$ & 4 & 2.8 \\
$36-40$ & 2 & 1.4 \\
40 y mayores & & \\
Escolaridad & 3 & 2.1 \\
Analfabeta & 14 & 9.6 \\
Sabe leer y escribir & 65 & $\mathbf{4 4 . 8}$ \\
Primaria & 34 & $\mathbf{2 3 . 4}$ \\
Secundaria & 29 & 20 \\
Bachillerato & 0 & 0 \\
Profesional & & 69.7 \\
Estado civil & 101 & $\mathbf{3 0 . 3}$ \\
Con pareja & 44 & 100 \\
Sin pareja & 145 & \\
\hline TOTAL & & \\
\hline
\end{tabular}

Fuente: $\mathrm{CRO}$

TABLA 2. ANTECEDENTES PERSONALES
PATOLÓGICOS
\begin{tabular}{|l|c|c|c|c|}
\hline VARIABLES & \multicolumn{2}{|c|}{$S i$} & \multicolumn{2}{c|}{ No } \\
\cline { 2 - 5 } & $f r$ & $\%$ & $f r$ & $\%$ \\
\hline Tuberculosis & 0 & 0 & 145 & 100 \\
Pulmonar & & & & \\
\cline { 1 - 1 } Diabetes Mellitus & 6 & 4.1 & 139 & 95.9 \\
\cline { 1 - 1 } HAS Crónica & 4 & 2.8 & 141 & 97.2 \\
\cline { 1 - 1 } Cirugía Pélvica/ & 37 & $\mathbf{2 5 . 5}$ & 108 & 74.5 \\
Uterina & & & & \\
\cline { 1 - 1 } Enfermedades & 0 & 0 & 145 & 100 \\
Inmunológicas & & & & \\
\hline
\end{tabular}

Fuente: CRO 
TABLA 3. SIGNOS QUE PRESENTARON LAS MUJERES EMBARAZADAS DURANTE LA VALORACIÓN

\begin{tabular}{|c|c|c|c|}
\hline SIGNOS & & $f r$ & $\%$ \\
\hline \multirow[t]{2}{*}{ Edemas en miembros pélvicos } & $\mathrm{Si}$ & 15 & 10.3 \\
\hline & No & 130 & 89.7 \\
\hline \multirow[t]{2}{*}{ Sangrados tras vaginales } & $\mathrm{Si}$ & 4 & 2.8 \\
\hline & No & 141 & 97.2 \\
\hline \multirow{2}{*}{ Anemia } & $\mathrm{Si}$ & 9 & 6.2 \\
\hline & No & 136 & 93.8 \\
\hline \multirow[t]{2}{*}{ Presión arterial } & $\mathrm{Si}$ & 6 & 4.1 \\
\hline & No & 140 & 96.6 \\
\hline \multirow[t]{2}{*}{ Cefalea } & $\mathrm{Si}$ & 30 & 20.7 \\
\hline & No & 115 & 79.3 \\
\hline Ardor al orinar & $\mathrm{Si}$ & 9 & 6.2 \\
\hline Crisis convulsivas & No & 136 & 93.8 \\
\hline \multirow[t]{2}{*}{ Mal nutridas/obesidad } & & $\begin{array}{c}0 \\
145\end{array}$ & $\begin{array}{c}0 \\
100\end{array}$ \\
\hline & & $\begin{array}{c}15 \\
130\end{array}$ & $\begin{array}{l}\mathbf{1 0 . 3} \\
89.7\end{array}$ \\
\hline
\end{tabular}

Fuente: CRO
De los antecedentes gineco-obstétricos, el $55.9 \%$ de las gestantes eran primigestas o multigestas de $>$ de $3 \mathrm{em}$ barazos, así mismo a $24.8 \%$ les habían practicado al menos una cesárea y solo el 4.9 \% había tenido productos de menos de $2500 \mathrm{gr}$. o mayores de $4000 \mathrm{gr}$ (Tabla 4). El $2.1 \%$ planteó haber presentado preeclampsia durante el embarazo anterior, el $20 \%$ presentó un peso mayor de 80 $\mathrm{Kg}\left(\mathrm{IMC}>\right.$ de $30 \mathrm{Kg} / \mathrm{m}^{2}$ ) y el $31 \%$ de las participantes en el estudio no se habían aplicado la vacuna antitetánica (Tabla 5). Por otro lado, al realizarles el examen clínico, ninguna de las participantes presentó alteración en su embarazo actual (Tabla 6).

La tabla 7 muestra que del total de las embarazadas que acudieron a consulta prenatal entre enero y marzo de 2016, que aceptaron participar en el estudio, el $69 \%$ es clasificada como de bajo riesgo por presentar una calificación de 0-5 puntos mientras el resto (31\%) mostró una puntación de $>6$ puntos que las identifica como de alto riesgo obstétrico, de acuerdo a los indicadores del cuestionario de factores de riesgo obstétrico de la Secretaría de Salud.

De igual forma, se puede observar que del total de mujeres gestantes, el grupo de jóvenes son las que presentan mayor riesgo obstétrico, bajo y alto, el $33.1 \%$ y el $12.4 \%$ respectivamente, mientras que las mujeres adolescentes (22.1\%) el bajo y alto riesgo estuvo presente en el $17.9 \%$ y $4.1 \%$ respectivamente.

\section{DISCUSIÓN}

Los resultados del presente estudio muestran un aspecto importante a considerar, la edad de las mujeres embarazadas, el $26.8 \%$ de la muestra seleccionada fueron adolescentes (de 18 años y menos) de las cuales solo el $17.9 \%$ presentaron alto riesgo obstétrico, mientras que el $82 \%$ fueron consideradas con bajo riesgo, condición que coincide con lo planteado por Tapia y otros ${ }^{(8)}$, en un estudio sobre perfil obstétrico de adolescentes embarazadas de un hospital público, desarrollado en la Ciudad de México, menciona que la mayoría de los adolescentes que participaron en el estudio se clasificaron como bajo riesgo obstétrico, asociado a factores sociales como la pobreza más que con la simple edad materna.

Respecto al nivel de escolaridad se observó que el $68.2 \%$ de las participantes en el estudio sólo cuentan con el nivel básico de educación, lo que se considera una limitante para tener conocimiento de su cuerpo y de los hechos básicos de la reproducción, así como la falta de conocimientos de medidas de prevención del embarazo y/o cuidado del mismo ${ }^{(8)}$.

Del estado civil de las mujeres embarazadas, los resultados del estudio muestran que el $30.3 \%$ no tienen pareja, al respecto Távara-Orozco y otros, ${ }^{(9)}$ en un estudio 


\section{TABLAS}

\begin{tabular}{|c|c|c|c|c|}
\hline \multicolumn{5}{|c|}{ TABLA 4. ANTECEDENTES GINECOBSTETRICOS } \\
\hline \multirow{2}{*}{ VARIABLES } & \multicolumn{2}{|c|}{ Si } & \multicolumn{2}{|c|}{ No } \\
\hline & fr & $\%$ & $f r$ & $\%$ \\
\hline Infertilidad & 1 & 0.7 & 144 & 99.3 \\
\hline $\begin{array}{l}\text { Primigestas o multigestas } \\
\text { ( }>\text { de } 3 \text { embarazos) }\end{array}$ & 81 & 55.9 & 64 & 44.1 \\
\hline Aborto previo & 17 & 11.7 & 128 & 88.3 \\
\hline Cesárea previa & 36 & 24.8 & 109 & 75.2 \\
\hline Dos o más cesáreas & 16 & 11 & 129 & 89 \\
\hline $\begin{array}{l}\text { Período intergenésico } \\
\text { corto }\end{array}$ & 7 & 4.8 & 138 & 95.2 \\
\hline ( < 2 años) & & & & \\
\hline Óbito (1) & 1 & 0.7 & 144 & 99.3 \\
\hline $\begin{array}{l}\text { Pérdida repetida de la } \\
\text { gestación }(<\text { de } 2)\end{array}$ & 0 & 0 & 145 & 100 \\
\hline $\begin{array}{l}\text { RN Muerto a la 1er. Se- } \\
\text { mana de VEU }\end{array}$ & 0 & 0 & 145 & 100 \\
\hline $\begin{array}{l}\text { Con peso menor de } 2500 \\
\text { gr. }\end{array}$ & 4 & 2.8 & 141 & 97.2 \\
\hline $\begin{array}{l}\text { Con peso mayor de } 4000 \\
\text { gr. }\end{array}$ & 3 & 2.1 & 142 & 97.9 \\
\hline
\end{tabular}

TABLA 5. EMBARAZO ACTUAL

\begin{tabular}{|l|c|c|c|c|}
\hline \multirow{2}{*}{ VARIABLES } & \multicolumn{2}{|c|}{ Si } & \multicolumn{2}{c|}{ No } \\
\cline { 2 - 5 } & $f r$ & $\%$ & $f r$ & $\%$ \\
\hline $\begin{array}{l}\text { Preclampsia en embara- } \\
\text { zo anterior }\end{array}$ & 3 & 2.1 & 142 & 97.9 \\
$\begin{array}{l}\text { Con peso mayor de 80 } \\
\text { kg. (IMC > 30 Kg/m } \text { m }^{2}\end{array}$ & 29 & $\mathbf{2 0}$ & 115 & 79.3 \\
$\begin{array}{l}\text { Con peso menor de 40 } \\
\text { kg. }\end{array}$ & 0 & 0 & 145 & 100 \\
Talla < de 1.50 m. & 1 & 0.7 & 144 & 99.3 \\
Duda FUM & 1 & 0.7 & 144 & 99.3 \\
Sin inmunización antite- & 45 & $\mathbf{3 1}$ & 100 & 69 \\
tánica & & & & \\
Grupo sanguíneo RH ne- & 0 & 0 & 145 & 100 \\
gativo & & & & \\
HB < 10 mg/dl & 0 & 0 & 145 & 100 \\
VDRL + & 2 & 1.4 & 143 & 98.6 \\
IVU & 9 & 6.2 & 136 & 93.8 \\
Embarazo múltiple & 0 & 0 & 145 & 100 \\
Fuente: CRO & & & $\mathbf{n}=145$
\end{tabular}




\begin{tabular}{|c|c|c|c|c|}
\hline \multicolumn{5}{|c|}{ TABLA 6. EXAMEN CLÍNICO ANORMAL } \\
\hline \multirow[t]{2}{*}{ VARIABLES } & \multicolumn{2}{|c|}{ Si } & \multicolumn{2}{|c|}{ No } \\
\hline & $f r$ & $\%$ & $f r$ & $\%$ \\
\hline $\begin{array}{l}\text { Exploración de mamas (anor- } \\
\text { mal), mastopatia fibroquística, } \\
\text { fibroadenoma mamario, cáncer }\end{array}$ & 0 & 0 & 145 & 100 \\
\hline $\begin{array}{l}\text { Exploración de pelvis (anor- } \\
\text { mal), malformación, condiloma } \\
\text { gigante, tumoraciones }\end{array}$ & 0 & 0 & 145 & 100 \\
\hline $\begin{array}{l}\text { Exploración cervical (Papani- } \\
\text { colaou o colposcopia anormal) }\end{array}$ & 0 & 0 & 145 & 100 \\
\hline T/A mayor de 130/90 & 0 & 0 & 145 & 100 \\
\hline $\mathrm{FCF}<120 \mathrm{lpm}$ & 0 & 0 & 145 & 100 \\
\hline $\mathrm{FCF}>160 \mathrm{lpm}$ & 0 & 0 & 145 & 100 \\
\hline $\begin{array}{l}\text { Variaciones mayores de } 25 \mathrm{lpm} \\
\text { sin actividad uterina }\end{array}$ & 0 & 0 & 145 & 100 \\
\hline
\end{tabular}

Fuente: CRO

TABLA 7. CLASIFICACIÓN DE RIESGO OBSTÉTRICO POR EDAD

\begin{tabular}{|c|c|c|c|c|c|c|}
\hline \multirow{3}{*}{$\begin{array}{c}\text { EDAD } \\
\text { (en años) }\end{array}$} & \multicolumn{4}{|c|}{$\begin{array}{c}\text { Clasificación de Riesgo } \\
\text { Obstétrico }\end{array}$} & \multirow{2}{*}{\multicolumn{2}{|c|}{ Total }} \\
\hline & \multicolumn{2}{|c|}{ Bajo riesgo } & \multicolumn{2}{|c|}{ Alto riesgo } & & \\
\hline & $f x$ & $\%$ & $f x$ & $\%$ & $f x$ & $\%$ \\
\hline 18 y menos & 26 & $17.9 \%$ & 6 & $4.1 \%$ & 32 & $22.1 \%$ \\
\hline $19-25$ & 48 & $33.1 \%$ & 18 & $12.4 \%$ & 66 & $45.5 \%$ \\
\hline $26-30$ & 14 & $9.7 \%$ & 7 & $4.8 \%$ & 21 & $14.5 \%$ \\
\hline $31-35$ & 10 & $6.9 \%$ & 10 & $6.9 \%$ & 20 & $13.8 \%$ \\
\hline $36-40$ & 2 & $1.4 \%$ & 2 & $1.4-5$ & 4 & $2.8 \%$ \\
\hline $41+$ & 0 & $0 \%$ & 2 & $1.4 \%$ & 2 & $1.4 \%$ \\
\hline Total & 100 & $69 \%$ & 45 & $31 \%$ & 145 & $100 \%$ \\
\hline \multicolumn{2}{|c|}{ Fuente: CRO } & \multicolumn{5}{|c|}{$\mathbf{n}=145$} \\
\hline
\end{tabular}


realizado en 2005 sobre características maternas de la preeclampsia y eclampsia, encontraron que en las mujeres embarazadas solteras aumentaba la posibilidad de presentar riesgo obstétrico con relación a las gestantes casadas, lo que indica que a la desprotección social y familiar en dichas mujeres, se le añade el estrés y quizás la violencia, situación existente en el contexto latinoamericano.

Dentro de los signos de alarma que presentaron las mujeres embarazadas, el ardor al orinar podría sugerir infecciones urinarias y que, a diferencia de lo encontrado por Calderón y otros, (2) en un estudio sobre presencia frecuente de infecciones en vía urinarias en mujeres embarazadas (el $53 \%$ de los casos), los resultados del estudio muestran que solo $6.2 \%$ presenta esta situación.

Es importante señalar que, del grupo en estudio, el $20 \%$ de las gestantes presentan un peso mayor de $80 \mathrm{Kg}$ (IMC $>30 \mathrm{Kg} / \mathrm{m}^{2}$ ), lo que en mujeres embarazadas se considera como obesidad leve ${ }^{(10)}$ y que se relaciona con lo obtenido en un estudio donde se estudiaron los factores de riesgo que se asociaron con más frecuencia a la preeclampsia, donde la obesidad ocupa el tercer lugar con una incidencia del $19.7 \%{ }^{(11)}$.

Más de la mitad de las gestantes (55.9\%) son primigestas y/o multigestas con más de tres hijos y de estas últimas casi el $25 \%$ tienen cesárea previa, lo que concuerda con lo encontrado en un estudio de casos y controles que determinó la relación entre factores de riesgo con el parto pretérmino, donde la incidencia de cesáreas en el grupo de casos fue del $25 \%(p=0.5)$ y más del $50 \% \quad(p>0.4)$ de las mujeres fue primípara o multípara ${ }^{(12)}$, lo cual las coloca en un nivel de vulnerabilidad alto para presentar alguna complicación durante el embarazo, parto y puerperio que ponga en peligro su salud y la de su hijo.

Aunque el embarazo es una situación fisiológica, también un proceso potencialmente de riesgo a lo largo su desarrollo, independientemente de la edad de las mujeres gestantes y es clasificado como de bajo, medio y alto riesgo, este último, se puede entender como gestación en la que la madre y el hijo se encuentran en mayor riesgo de presentar signos de alarma que ponen en peligro sus vidas. Referente a la relación que existe entre la edad y el riesgo obstétrico, en este estudio se encontró que las mujeres entre 19 y 25 años son las mayormente afectadas por esta condición, lo cual difiere con lo planteado por otros investigadores que concluyen que el embarazo a edad temprana conlleva a complicaciones durante la gestación en el grupo de adolescentes de 13 a 18 años (13).

Sin embargo, en un estudio realizado en Morelos se encontró que de 94 casos de muertes maternas, la mayor parte de casos fue de mujeres entre los 19-24 años de edad (40\%), le siguió el grupo etario de 25-34 años
(36.37\%) y finalmente el grupo de 35 años o más con un $23.3 \%^{(14)}$, es importante destacar la relación que existe entre lo encontrado en este estudio, donde el grupo que tiene un mayor riesgo obstétrico es el de mujeres entre 19-25 años (12.4\%), y que es en este grupo de edad donde se debería enfatizar la importancia de la promoción de la salud obstétrica, sobre todo en aquellas usuarias que presentan antecedentes de complicaciones graves en embarazos previos.

\section{CONCLUSIONES}

En las últimas décadas, la muerte materna ha cobrado importancia en la salud de nuestro país y a nivel mundial. Son muchas las repercusiones que impacta la salud materna, entre los cuales se han considerado de mayor relevancia los de tipo económicos y sociales cuando se trata de salud pública, ya que la falta de recursos para una adecuada atención puede impactar de forma negativa en la salud de las futuras madres mexicanas que no cuentan con el apoyo para llevar un adecuado control prenatal.

En cuanto a las mujeres embarazadas que acuden a consulta prenatal al Centro de Salud, se observó que, de las participantes en estudio, las mujeres jóvenes (19 a 25 años) son las que presentan mayor riesgo obstétrico y no las madres adolescentes (18 años y menos) que formaban parte de la población estudiada.

Es importante señalar que entre menos controles prenatales se realicen las mujeres durante el embarazo es mayor el riesgo obstétrico que puedan presentar, lo cual se entiende porque la frecuencia y calidad del control prenatal permite hacer una evaluación integral de la salud de la madre y el hijo, que puede contribuir a la detección temprana de factores que compliquen el embarazo, parto y puerperio, poniendo en peligro la vida del binomio madre e hijo.

Es necesario proporcionarles información sobre el embarazo y sus complicaciones, para que sean capaces de identificar los factores de riesgos durante el embarazo, parto y puerperio y las consecuencias que estos tienen para su salud y la de su hijo.

También se considera de vital importancia que la información sobre riesgo obstétrico la reciban, no sólo quienes presentan problemas durante el embarazo, sino cualquier mujer que se encuentre en estado de gestación, como medida preventiva y así contribuir a la diminución de la morbimortalidad materno infantil.

Es conveniente considerar no sólo el enfoque biomédico, sino tomar en cuenta los factores sociales y económicos de las madres embarazadas, ya que la mayoría cuentan con pocos recursos, aunado a las escasas oportunidades laborales, que con el embarazo se limitan todavía más, favoreciendo con esto un círculo vicioso cada vez más arraigado de pobreza. 
Un elemento importante a considerar para la reducción de la morbimortalidad materna es la comprensión y consideración de los determinantes sociales para la salud que impactan en la salud materna y se asocian a la aparición de riesgos obstétricos en la mujer embarazada.

\section{Recomendaciones}

Es muy importante la impartición de charlas sobre planificación familiar durante la consulta externa, así como impartir programas de educación sobre salud reproductiva en preparatorias, para poder prevenir embarazo en adolescentes.

Aceptar a toda mujer embarazada que no cuente con algún tipo de seguro médico o que no pertenezcan al sector o área de afluencia, para atender a la mayor población de embarazadas posible y disminuir el número de embarazos sin vigilancia médica.

Crear grupos de auto ayuda de embarazadas que sirva para monitorearlas periódicamente y para ofrecerles herramientas de autocuidado que les permita identificar factores de riesgo que pongan en peligro su salud y la de su hijo.

\section{REFERENCIAS}

1. Secretaría de Salud. Norma Oficial Mexicana 007-SSA2-1993. 1993.

2. Calderón J, Vega G, Velázquez J, Morales R, Vega A. Factores de riesgo materno asociados al parto pretérmino. Revista Médica del IMSS. 2005; 43(4).

3. Organización Mundial de la Salud (OMS). Mortalidad materna. [Online].; 2016 [cited 2016 Noviembre 28. Available from: http://www.who.int/mediacentre/factsheets/fs348/es/.

4. Ruiz C. Restos de la mortalidad maternal en México. s/f..

5. Freyermuth G, Luna M, Muños J. Indicadores 2013. Objetivo del desarrollo del milenio 5: avances en México. Observatorio de mortalidad materna en México; 2015.

6. Álvarez M, Álvarez S, González G, Pérez D. Caracterización de la morbidlidad materna extremadamente grave. Revista Cubana de Higiene y Epidemiología. 2010; 48(3): p. 310-20.

7. Diario Oficial de la Federación. Ley General de Salud: reformada en 2016. 1984..

8. Tapia M, Lossi S, Pérez C, Jiménez M. Perfil obstétrico de mujeres embarazadas en hospital público: riesgo al inicio del trabajo de parto, parto y puerperio. Rev Layinno-Am.Enfermagem. 2015; 23(5): p. 829-36.

9. Távara-Orozco L, Orderique L, Zegarra-Samame T, Huamaní S, Félix F, Espinoza-Tarazona $\mathrm{K}$, et al. Reprecusiones maternas y perinatales de la violencia basada en género. Revista Peruana de Ginecología y Obstetricia. 2007; 53(1): p. 10-7.

10. Secretaría de Salud. Diario Oficial de la Federación [DOF]. [Online].; 2016 [cited 2015 Agosto. Available from: http://www.dof.gob.mx/nota_detalle. php?codigo $=5432289 \&$ fecha $=07 / 04 / 2016$.

11. García R, Llera A, Pacheco A, Delgado M, González A. Resultados maternos-perinatales de pacientes con preeclampsia. Revista Cubana de Obstetricia y Ginecología. 2012; 38(4): p. 467-77.

12. Chira J, Sosa J. Factores de riesgo para parto pretérmino en gestantes del hospital provincial docente de belen de lambayeque. Salud \& Vida Sipanense. 2015; 2(2): p. 7-18.

13. Domínguez-Hanaya R, Herazo-Beltrán M. Edad de la gestante adolescente como factor de riesgo para complicaciones en el embarazo.Cartagena (Colombia) 2009. Estudio de corte transversal. Revista Colombiana de Obstetricia y Ginecología. 2011; 62(2): p. 141-7.

14. Ordaz-Martínez K, Rangel R, Hernández-Girón C. Factores de riesgo asociados con mortalidad materna en el estado de. Ginecol Obstet Mex. 2010; 78(7): p. 357-64.

15. Observatorio de mortalidad materna en México. Indicadores de mortalidad materna. [Online].; 2013 [cited 2016 Febrero 9. Available from: http://omm. org.mx/index.php/indicadores-nacionales/indicadores.

16. Secretaría de Salud. Guía de Práctica Clínica para el control prenatal con enfoque de riesgo. 2009..

17. Ransom E, Yinger N. Por una maternidad sin riesgos: cómo superar los obstáculos en la atención a la salud materna Washington, D.C.: Population Reference Bureau; 2002. 